\title{
Safety analysis of energy storage station based on DFMEA
}

\author{
Xin $\mathrm{Li}^{1, \mathrm{a}}$, Qingshan Wang ${ }^{2}$, Yan $\mathrm{Chen}^{3}$, Yan $\mathrm{Li}^{3}$, Zhenyu $\mathrm{He}^{1}$, Tianqi Wang ${ }^{1}$ and Xijin $\mathrm{Wu}^{1}$ \\ ${ }^{1}$ Nari Research Institute, NARI Technology Co., Ltd., Nanjing, China \\ ${ }^{2}$ Economic and Technological Research Institute of Jiangsu Electric Power Company, Nanjing, China \\ ${ }^{3}$ Jiangsu Academy of Safety Science and Technology, Nanjing, China.
}

\begin{abstract}
In order to ensure the normal operation and personnel safety of energy storage station, this paper intends to analyse the potential failure mode and identify the risk through DFMEA analysis method, and then through the targeted treatment of potential risk items, formulate effective design prevention countermeasures and personnel emergency measures, so as to improve the energy storage station. The reliability of the battery can reduce the safety risk and ensure the safe operation of energy storage station.
\end{abstract}

\section{Introduction}

The safety of lithium-ion battery storage power station is a major problem that needs the alarm bell to ring for a long time ${ }^{[1-3]}$. With the research and development of new materials, the innovation of battery manufacturing technology and the participation of many scientific research institutions and enterprises, the performance of lithium-ion battery is improving rapidly, and the safety performance of battery is also greatly improved ${ }^{[4-6]}$. However, as shown in Figure 1, local thermal runaway phenomenon is easy to occur in the use process. South Korea has encountered the crisis of energy storage power station fire. The 21 energy storage fire incidents in South Korea since 2017 have brought about the overall stagnation of South Korea's local energy storage industry. By analysing the past 21 fires at energy storage plants, 16 fires were reported to have been caused by battery systems. In 2019, a large-scale battery energy storage project exploded at the public service utility company (APS) in West Valley, Arizona. ${ }^{[7]}$.

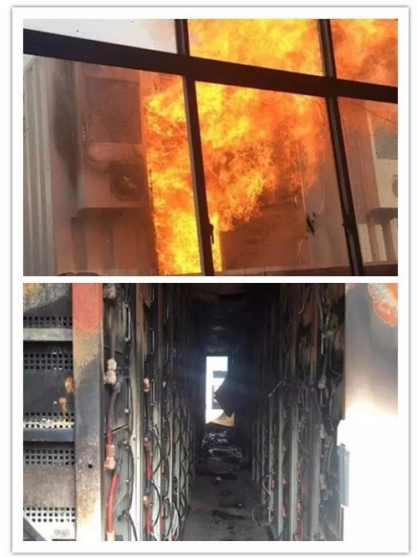

Figure 1 Thermal runaway phenomenon of energy storage station

It is very important for the safe operation of the energy storage system to study the fire warning technology of Li-ion battery energy storage power station ${ }^{[10]}$. The recognition of thermal runaway and thermal diffusion characteristics of lithium-ion batteries is discussed. In order to ensure the normal operation and personnel safety of energy storage power station, this paper intends to analyse the potential failure mode and identify the risk through DFMEA analysis method, and then through the targeted treatment of potential risk items, formulate effective design prevention countermeasures and personnel emergency measures, so as to improve the reliability of energy storage lithium ion battery module and reduce the safety risk and ensure the safe operation of the energy storage power station ${ }^{[11]}$.

\section{DFMEA analyse methods}

Potential failure mode and effect analysis (FMEA) was the first reliability analysis tool developed by American aerospace industry in 1960s. Using this tool, we can find and evaluate the potential failure modes of products or processes, point out the weaknesses and possible defects judged according to experience as early as possible, analyse the failure consequences and risks caused, and finally find measures to avoid or reduce these potential failures in the decision-making process.

DFMEA (Design Failure Mode Effect Analysis) failure mode impact analysis is to analyse the potential failure modes of each component unit of the product and the functional impact on the product in the process of system or equipment design. Each potential failure mode is classified according to its severity, and corresponding preventive and improvement measures are proposed to improve the reliability of the system or equipment. It is the basis of DFMEA analysis method.

DFMEA can achieve the following objectives in the process of system or equipment design:

1. Find out what is important to system function and performance. All kinds of affected components and their

\footnotetext{
a Corresponding author: lixin11@sgepri.sgcc.com.cn
} 
failure modes are found out and their influence degree analysed.

2. Be able to ensure that all failure modes and effects of all components of the system are carefully considered and relevant measures are taken, which can help designers and decision makers to select the best scheme to meet the system reliability requirements from various design schemes.

3.Make objective and scientific evaluation of relevant measures, testing equipment, etc. in design review.

4. Be able to provide basis and implementation conditions for quantitative analysis of system reliability design and provide system reliability evaluation results.

5. Provide data, technology, and conditions for further improvement of system design.

6. Accumulate technical data for the next similar new product design scheme selection and decision-making.

DFMEA has two basic analysis methods which called hardware method and function method. Function method that can be completed by each product can be classified according to the output. This method lists the outputs one by one and analyses their failure modes. When the product composition is not clear, or the product complex requirements are analysed from the initial agreement level down, that is, the top-down analysis generally adopts the functional method.

Hardware method needs to analyse each failure mode according to product function, and list each department with table, meanwhile, the failure mode, failure mechanism and impact are analysed. When the product can be clearly determined according to the design drawings and other engineering data, the hardware method is generally used. This is a more rigorous method. This method is applicable to the analysis from component level to system level, from bottom to top, or from any level to the next level.

DFMEA of fire warning technology of Li-ion battery energy storage power station is listed as follows.

\subsection{Capacity decay failure}

As related systems mentioned that, during the standard cycle life test, when the number of cycles reaches 500, the discharge capacity shall not be less than $90 \%$ of the initial capacity. Or when the number of cycles reaches 1000 , the discharge capacity shall not be less than $80 \%$ of the initial capacity. If the capacity drops sharply within the standard cycle range, it belongs to capacity attenuation failure. The failure of battery capacity attenuation is caused by the failure of materials, which is closely related to the objective factors such as battery manufacturing process and battery use environment. From the material point of view, the main causes of the failure are the structural failure of the positive material, the transition growth of SEI on the negative surface, the decomposition and modification of the electrolyte, the corrosion of the collecting fluid, and the trace impurities in the system.

\subsection{Structural failure of positive material}

Structural failure of positive material includes particle breakage, irreversible phase transformation, material disorder, etc. During the charging and discharging process, the structure of $\mathrm{LiMn}_{2} \mathrm{O}_{4}$ will be distorted due to Jahn teller effect, and even the particles will be broken, resulting in the failure of electrical contact between particles. During the charging and discharging process of $\mathrm{Li}_{1.5} \mathrm{~N}_{\mathrm{i} 0.5} \mathrm{O}_{4}$ material, the phase transition of tetragonal system cubic system will take place. During the charging and discharging process of $\mathrm{LiCoO}_{2}$ material, due to the transition of $\mathrm{Li}$, Co will enter the $\mathrm{Li}$ layer, resulting in the disorder of the layered structure and restricting its capacity.

\subsection{Negative electrode material failure}

The failure of graphite electrode mainly occurs on the surface of graphite. The surface of graphite reacts with electrolyte to produce solid electrolyte interface phase (SEI). If excessive growth leads to the decrease of lithium-ion content in the internal system of battery, the result is capacity degradation. The failure of silicon anode materials is mainly due to the cyclic performance problems caused by its huge volume expansion. The formation of nano voids between the stripped Li and SEI films is attributed to the accumulation of lithium metal vacancies. The rapid dissolution of lithium leads to the growth and aggregation of voids, followed by the collapse of SEI.

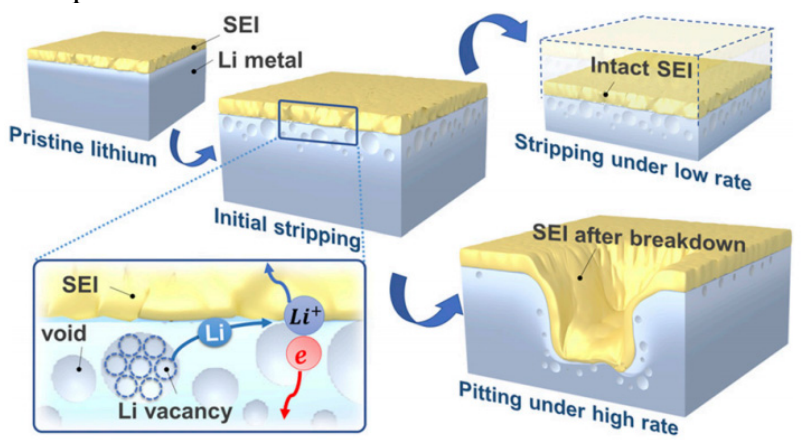

Figure 2 Disintegration mechanism of SEI

\subsection{Electrolyte failure}

The stability of $\mathrm{LiPF}_{6}$ is poor, and it is easy to decompose to reduce the content of $\mathrm{Li}+$ in the electrolyte. It is also easy to react with trace water in the electrolyte to generate HF, which causes corrosion inside the battery. The poor air tightness results in the deterioration of electrolyte, the change of viscosity and chroma of electrolyte, and the sharp decline of ion transport performance.

\subsection{Failure of current collector}

Corrosion of current collector and decrease of adhesion of collecting fluid. HF produced by the failure of the above electrolyte will cause corrosion to the collecting fluid and generate compounds with poor conductivity, 
resulting in increased ohmic contact or failure of active substances. In the process of charging and discharging, the copper foil is dissolved at low potential and deposited on the positive electrode surface, which is called "copper evolution". The common form of collector failure is that the binding force between collector and active substance is not enough, which leads to the stripping of active substance and cannot provide capacity for battery.

\subsection{Increased internal resistance}

The increase of internal resistance of lithium battery will be accompanied by the decrease of energy density, voltage and power, and heat generation. The main factors that lead to the increase of internal resistance of Li-ion battery are the key materials and the operating environment of the battery.

\subsection{Key materials of battery}

Microcracks and breakage of positive materials, destruction of negative materials and over thickness of SEI on the surface, aging of electrolyte, separation of active substances from collecting fluid, poor contact between active substances and conductive additives (including loss of conductive additives), blocking of diaphragm shrinkage, abnormal welding of cell ears, etc.

\subsection{Battery use environment}

High/low ambient temperature, overcharge and over discharge, high-rate charge and discharge, manufacturing process and battery design structure, etc. The generation of heat and gas are two common characteristics in the process of overcharge. The heat comes from ohmic heat and side effects. Firstly, lithium dendrites grow on the anode surface due to excessive lithium intercalation. The growth time of lithium dendrite is determined by the stoichiometric ratio of cathode and anode. Secondly, the excessive extraction of lithium leads to the collapse of cathode structure due to heating and oxygen release. The release of oxygen accelerates the decomposition of electrolyte and produces a large amount of gas. As the internal pressure increases, the exhaust valve opens and the battery begins to exhaust. After the active substance in the cell contacts with air, it reacts violently and releases a lot of heat. Overcharge protection can be carried out from voltage management and material adjustment. During the over discharge period, the battery with the lowest voltage in the battery pack can be forced to discharge by other batteries connected in series. During the forced discharge, the pole reverses and the battery voltage becomes negative, which leads to the abnormal heating of the over discharge battery. The dissolved copper ions migrate through the membrane and form copper dendrites with low potential on the cathode side. With the increase of growth, copper dendrites may penetrate the membrane, resulting in severe thermal runaway.

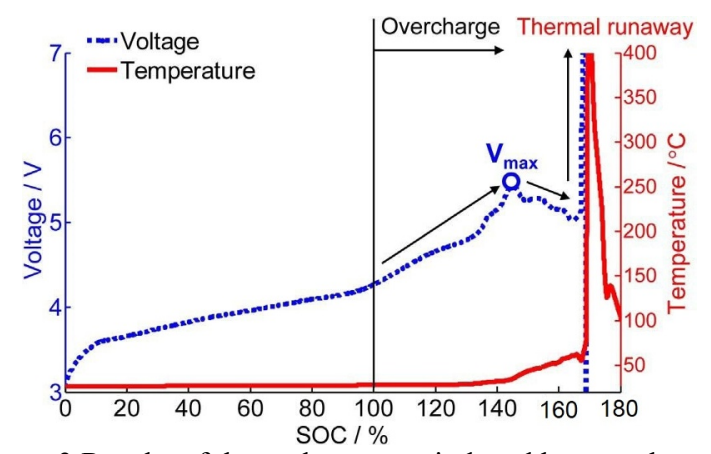

Figure 3 Results of thermal runaway induced by overcharge in commercial lithium-ion batteries

The changes of voltage, temperature and internal resistance during overcharge are shown in Figure 4. It can be roughly divided into four stages. In the first stage, there was no obvious side reaction inside the battery, and the temperature and internal resistance of the battery changed little. Moreover, the electrolyte was oxidized at the positive side, and lithium was precipitated on the surface of the negative electrode. The reaction between lithium and solvent makes SEI film thicker, the impedance of battery increases, and the temperature of battery begins to rise slowly. Then the temperature rise of the battery is accelerated, the battery bulges obviously, the electrolyte oxidation on the positive side accelerates, and a lot of heat and gas are released. Lithium continued to precipitate on the anode surface, and the SEI film began to decompose, and the lithium graphite reacted with the electrolyte. In the last stage, the internal pressure of the battery exceeded the limit, the shell cracked, the diaphragm contracted and deformed, and the battery heat was out of control.

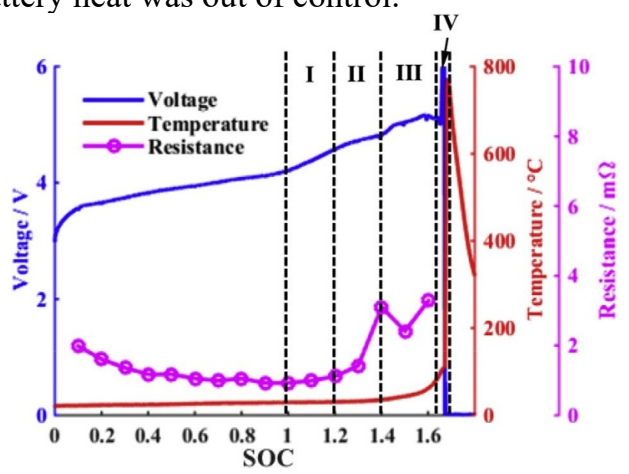

Figure 4 Results of thermal runaway induced by overcharge in commercial lithium-ion batteries

\subsection{Internal short circuit}

Short circuit can be separated by four reasons. Firstly, short circuit between copper / aluminium current collector. The contact of positive and negative collector caused by untrimmed metal foreign matters puncturing diaphragm or electrode, displacement of electrode piece or ear in battery packaging during battery production or use. Short circuit between copper and aluminium collector is caused by the positive and negative current collector contact caused by the unshaven metal foreign body puncturing the diaphragm or electrode during the production or use of the battery, and the displacement of the electrode piece or ear in the battery package. Furthermore, short circuit caused by diaphragm failure. 
The aging, collapse and corrosion of the separator will lead to diaphragm failure. The failure diaphragm will lose electronic insulation, leading positive and negative micro contact, and then local heating will occur. Continuous charging and discharging will spread around, resulting in thermal loss control. Thirdly, if the transition metal impurities in the positive slurry are not removed, it will lead to piercing the diaphragm or lead to the formation of lithium dendrite in the negative electrode, leading to internal short circuit. Finally, the lithium dendrite will appear in the place with uneven local charge during the long cycle, and the dendrite will cause internal short circuit through the diaphragm. In the process of battery design and manufacture or battery pack assembly, unreasonable design or excessive local pressure will also lead to internal short circuit. Internal short circuit will also occur under the guidance of battery overshoot and over discharge.

\section{Potential failure analysis}

\section{consequence}

\subsection{Lithium-ion battery failure mode}

The consequence of potential failure is the influence of failure mode on the operation, personnel safety, and product function of energy storage station. The consequences of failure should be described according to the circumstances that may be found or experienced. The potential failure mode for the hidden danger of electric collision of energy storage station. The results of potential failure consequence analysis are shown in Table 1.

Table 1. Lithium-ion battery failure mode and effect analysis

\begin{tabular}{|c|c|c|}
\hline Failure reason & Influence & $\begin{array}{c}\text { Performance } \\
\text { degraded }\end{array}$ \\
\hline $\begin{array}{c}\text { Structural failure } \\
\text { of positive } \\
\text { material }\end{array}$ & $\begin{array}{c}\text { Lithium loss and } \\
\text { increases internal } \\
\text { resistance }\end{array}$ & Capacity \\
\hline $\begin{array}{c}\text { Structural failure } \\
\text { of negative } \\
\text { material }\end{array}$ & $\begin{array}{c}\text { Lithium loss and } \\
\text { increases internal } \\
\text { resistance }\end{array}$ & Capacity \\
\hline $\begin{array}{c}\text { Electrolyte failure } \\
\text { Increased internal } \\
\text { resistance }\end{array}$ & $\begin{array}{c}\text { Increases internal } \\
\text { resistance }\end{array}$ & Stability \\
decreased & Capacity and \\
\hline $\begin{array}{c}\text { Battery use } \\
\text { environment }\end{array}$ & $\begin{array}{c}\text { Stability } \\
\text { decreased }\end{array}$ & $\begin{array}{c}\text { Capacity and } \\
\text { energy }\end{array}$ \\
\hline $\begin{array}{c}\text { Internal short } \\
\text { circuit }\end{array}$ & $\begin{array}{c}\text { Stability } \\
\text { decreased }\end{array}$ & $\begin{array}{c}\text { Capacity and } \\
\text { energy }\end{array}$ \\
\hline
\end{tabular}

\subsection{Determine the current design control measures}

According to the structural characteristics of power battery system, electrical safety failure mode, the content of failure mode analysis needs to be reflected in the design scheme in the product design process, and the failure can be eliminated through the design process.
During the design of the energy storage station, the following electrical safety design requirements are put forward: (1) In addition to the capacity separation method, the separation methods such as internal resistance, AC impedance spectrum and so on should be increased. (2) Strengthen the monitoring of battery inconsistency in battery pack. (3) Control the temperature of energy storage station and add smoke alarm device in battery box.

\section{Conclusion}

Through the analysis of the above specific examples, the analysis methods and concepts of DFMEA are properly and flexibly transformed, and the risk identification and analysis are carried out for the hidden danger of electric collision of energy storage station, and the targeted design preventive measures and emergency measures for operators are formulated for the risk points, and these measures are respectively applied to the design and development, operation manual, emergency repair regulations, daily maintenance specifications, etc. The effective implementation and application of these measures provide a great guarantee for the safe operation of energy storage station.

\section{Acknowledgments}

This work was financially supported by State Grid Science and Technology fund (5400-201940486A-0-000 ) and Nation Science Foundation of China (51808266).

\section{References}

1. Chunli W., Limiao G., Ping K., Yechao T., Mingming L. I., Energy Storage ence and Technology (2018)

2. Zhang L., Zhao P., Xu M., Wang X., Applied Energy 261(2020)

3. Shouping X. U., Chaoyong H., Juan H. U., Huanling W, Electric Power Construction 35.5(2014):72-78

4. 3Rafiz K., Lin J. Y. S, Sustainable Energy \& Fuels 4(2020)

5. Xu T., Zhou C., Zhou H., Wang Z., Ren J, Chinese Journal of Chemistry (2019)

6. Iretomiwa E., Krishna S., Ankur J, Applied Thermal Engineering 145(2018)

7. Chombo P. V.,Laoonual Y., Journal of Power Sources 478(2020)

8. Zeng Z., Murugesan V., Han K. S., Jiang X., Cao Y., Xiao, L, Nature Energy (2018)

9. Lingxi K., Chuan L., Jiuchun J., Michael P, Energies 11.9(2018):2191

10. Hao, H. E. , et al. Energy Storage ence and Technology (2019).

11. Schlasza C., Ostertag P., Chrenko D., Kriesten R., Bouquain D, ITEC (2014) 